\title{
Parkinson's Disease - Levodopa Complications
}

\author{
David B. King
}

\begin{abstract}
Parkinson's disease is a readily recognized clinical syndrome, characterized by resting tremor, rigidity, bradykinesia and loss of postural reflexes. The introduction of levodopa transformed our management of this disease. As our experience has increased the long term side effects of levodopa have become more apparent. Levodopa complications comprise: wearing off, motor fluctuations, and psychiatric complications. The complexity of these clinical phenomena has been worked out with time and is now well-recognized. A number of management strategies can ameliorate these long-term complications. This article reviews the current management of late-stage Parkinson's disease.
\end{abstract}

R SUM : Maladie de Parkinson - Complications du traitement par la l vodopa. La maladie de Parkinson est un syndrome clinique facilement reconnaissable, caract ris par un tremblement de repos, de la rigidit, de la bradykin sie et une perte des $r$ flexes posturaux. L'introduction de la 1 vodopa a transform le traitement de cette maladie. Avec l'exp rience, les effets secondaires long terme de la 1 vodopa sont devenus plus vidents. Les complications du traitement par la 1 vodopa incluent: l' puisement de l'effet en fin de dose, les fluctuations motrices et les complications psychiatriques. La complexit de ces ph nom nes cliniques est maintenant mieux comprise et mieux reconnue. Certaines strat gies th rapeutiques peuvent am liorer ces complications du traitement long terme. Cet article revoit le traitement de la maladie de Parkinson en phase avanc e.

Can. J. Neurol. Sci. 1999; 26: Suppl. 2-S13-S20

Voltaire once said, "Doctors are men who prescribe medicines of which they know little, to cure diseases of which they know less in human beings of whom they know nothing." His criticism cannot be taken lightly. We might argue, in our defence, that we have made substantial progress since his time.

Parkinson's disease is a clinical syndrome characterized by resting tremor, rigidity, bradykinesia, and loss of postural reflexes. Evidence indicates that diminished striatal dopamine neurotransmission is a sufficient condition for its development. ${ }^{1}$

The introduction of levodopa transformed our management of this devastating disease. It is difficult to overestimate the impact which the introduction of levodopa had on the treatment of Parkinson's disease. Cotzias, ${ }^{1}$ shortly after its introduction, described the improvement of motor function as "dramatic". There was little question, in those early halcyon days, as to which medication to use in symptomatic Parkinson's disease. Initial complications of therapy included nausea, vomiting, postural hypotension and abnormal involuntary movements. These complications arose because of the poor therapeutic index of levodopa and the necessity to use maximum tolerated doses in the early studies. The introduction of combination therapy with carbidopa or benserazide and the slow introduction of the drug largely obviated these initial side effects. The dramatic benefits were not to be sustained. The complications of late therapy have become ever more apparent. Our early therapy choices have come under detailed scrutiny. ${ }^{2}$ This brief review will examine those late complications.

\section{EPIDEMIOLOGY}

About $10 \%$ of patients each year will develop motor fluctuations and dyskinesias, either as a result of the advancement of the Parkinson's disease or as a result of levodopa therapy. ${ }^{3}$

The beneficial early response in Parkinson's disease continues generally for about five years. Patients then will begin to move from a stable state through a succession of changes commencing with wearing off, subsequently dyskinesias and then onoff problems.

Block and his colleagues in the CR First Study followed 618 patients with non-fluctuating Parkinson's disease. ${ }^{4}$ Approximately half the patients were put on Sinemet CR and half on Sinemet. After five years, some $20 \%$ had increased "Off" time or dyskinesia. This paper is one of the most optimistic in terms of sustained benefit from levodopa therapy. Other papers have been far more pessimistic, indicating that approximately onethird of patients are deteriorating and one-third have significant dyskinesias and fluctuations of performance at five years. Miyawaki et al. ${ }^{5}$ in a retrospective study of some 811 patients showed that only $30 \%$ were free of fluctuations at 15 years.

Reprints request to: D.B. King, 5523 Spring Garden Road, Suite 208, Halifax, Nova Scotia, Canada B3J 3T1 


\section{OVERVIEW}

\section{Levodopa Complications}

There are a number of late complications that occur in Parkinsonian patients on levodopa therapy. These follow a more or less predictable time course. We may list them for convenience.

1. Wearing off

2. Motor fluctuations

3. Psychiatric complications

There are a number of fluctuations that occur in Parkinson's disease which are unrelated to drug therapy and these include:

1. Kinesia paradoxica

2. Freezing

3. Sleep benefit

4. Tremor enhancement by emotional stress

In early disease, the therapeutic response is predictable. In fact, the response to levodopa is generally linear, so that as the dose is increased, the patient continues to show signs of improvement to a point. Beyond a certain dose, even in early disease, the motor and psychiatric symptoms characteristic of late complications will develop.

Unfortunately, as time advances, a single dose of levodopa fails to sustain motor performance between doses. A single dose, if sufficiently high, tends to produce a peak dose dyskinesia, though the time course of benefit remains much the same. With advance of the disease the same levodopa dose tends to produce a more limited beneficial response. Dyskinesias become more prominent and more prolonged. As the disease worsens, a single dose tends to produce dyskinesia before the patient turns "On". There is a short window of benefit, often contaminated by peak dose dyskinesia, and then dyskinesia as the dose wanes. This pattern of dyskinesias before and after the "On" phase is referred to as diphasic. Higher doses tend to produce a slight prolongation of benefit, though the complications of diphasic and peak-dose dyskinesia remain and worsen. Fluctuations from mobility to immobility occur more frequently and become more abrupt and more disabling. ${ }^{6}$

It is unclear as to the fate of administered levodopa. It may be that the levodopa enters the pre-synaptic dopaminergic neuron and is converted to dopamine which is stored in vesicles, ready for release, a physiologic pattern. On the other hand, levodopa may enter the pre-synaptic neuron but be stored in extra-vesicular storage areas which result in a slow uncoupled release of the compound. A third possibility is that it is stored in non-dopaminergic neurons, perhaps intra-neurons, which also results in a slow, uncoupled release. Regardless of the exact mechanism it is likely that nigral cells continue to die and their abilities to convert levodopa to dopamine, store dopamine and release it are progressively compromised. This lack of a storage facility may account for the end-of-dose deterioration.

Changes clearly occur post-synaptically in the striatum as a result of denervation. The dopamine agonist apomorphine acts post-synaptically, independent of presynaptic mechanisms. The duration of the antiparkinsonian response after discontinuation of an optimal-dose infusion declines with disease progression. Disease severity alters the response to dopaminergic stimulation in the striatum. The reason for these post-synaptic changes are unknown.
The reasons for the central changes in dopamine metabolism have not been fully elucidated. They may be simply due to disease progression or may be due to levodopa toxicity. There is evidence on both sides of this issue. The clinical data support the non-toxicity of levodopa. ${ }^{7,8}$ The theoretic and animal data suggest possible mechanisms for this toxicity to occur. Agid ${ }^{9}$ has recently reviewed the complex question of levodopa toxicity and concluded that it was unlikely that levodopa was toxic. The clinical studies using dopamine agonist therapy early and adding smaller doses of levodopa later suggest a better long term response with less fluctuations. ${ }^{10}$

There may be peripheral factors, outside of the nervous system, contributing to the fluctuations. These peripheral factors include the absorption of levodopa from the gut, the breakdown of levodopa outside the central nervous system and the transfer of levodopa across the blood brain barrier. While there is evidence that the peripheral metabolism of levodopa does not change with time, ${ }^{11}$ it is likely that the peripheral metabolism becomes more important as the handling of central levodopa becomes less buffered and efficient.

There are, therefore, post-synaptic and pre-synaptic changes in the nigrostriatal system along with peripheral factors that are important in the development of the late complications of levodopa therapy.

\section{Wearing Off Phenomenon}

Most Parkinsonian patients taking levodopa in the conventional fashion of three to four times per day, initially experience more or less constant benefit throughout the day. Wearing off or end-of-dose deterioration is usually the earliest manifestation of fluctuations in Parkinson's disease. It is the re-emergence of Parkinsonism three hours or so after a dose of levodopa. With time, the duration of the "On" response lessens and the wearing off phenomenon becomes more prominent. Each dose lasts for progressively shorter periods. The pattern of response becomes sinusoidal: improvement followed by worsening.

This may be manifest as early morning akinesia. Patients are often profoundly disabled immediately upon awakening in the morning, having been without medication for several hours. During the night turning over in bed becomes progressively more difficult, sialorrhoea is troublesome and they may have great difficulty in swallowing the first daily dose of drug.

This response can occur in as many as $66 \%$ of patients after six years of treatment with a levodopa preparation. ${ }^{12}$

As Parkinson's disease progresses there is a decline both in 1aromatic amino acid decarboxylase and intraneuronal vesicular storage sites for dopamine in the striatum. This progressive death of cells in the substantia nigra and thus their ability to synthesize, re-uptake, and store dopamine was hypothesized to represent the basis for wearing off. ${ }^{6}$ Rat data support this theory. ${ }^{13}$ Fluctuating patients given ${ }^{18}$ F6-fluorodopa accumulated less fluorodopa and its metabolites in their striata than did non-fluctuating patients. ${ }^{14}$ Fabbrini et al. found that, following the abrupt cessation of steady-state levodopa infusions to fluctuating patients compared to stable patients, the efficacy half-life decreased and the initial efficacy decay slope increased in the fluctuators. ${ }^{15}$ Together these data suggest that end-of-dose deterioration arises from disease progression and the loss of nigral neurons. Further supporting this contention is the fact that patients in the "Off" period will 
respond to exogenous levodopa or apomorphine, a dopamine agonist. ${ }^{16}$

\section{Motor Fluctuations}

As time advances levodopa begins to induce a variety of abnormal movements. These may be conveniently summarized: 1. Hyperkinetic

Peak dose dyskinesia

Diphasic dyskinesia

Myoclonus

Restless leg syndrome

2. Dystonic

Early-morning dystonia

End-of-dose dystonia

Peak-dose dystonia

3. Hypokinetic

Akinetic freezing of gait
On-Off
Drug Failure

\section{Peak Dose Dyskinesia}

Peak dose dyskinesia is chorea occurring at the peak of levodopa effect. As time advances dyskinesias tend to occupy more and more of the "On" period and become progressively more severe. They are more prominent on the most involved side but with time they become generalized. They involve the face, axial structures and the limbs. They can reach the point of severity where they impair function. Characteristically, in the early stages, they do not bother the patient. It is often the caregiver who calls attention to them. The patients prefer them to the "Off" periods.

\section{Diphasic Dyskinesia}

These are dyskinesias that arise before the onset of the "On" period and recur as the patient is going "Off". They may occur at either end of the "On" period or both. These can be a particularly troublesome form of dyskinesia. They are seemingly dependent on the flux of levodopa. In one study, ${ }^{17}$ dyskinesias with repetitive alternating movements were exclusively associated with diphasic dyskinesias.

\section{Myoclonus}

Myoclonus occurs primarily in the legs and is usually nocturnal.

\section{Restless Leg Syndrome}

Despite the fact that levodopa is often used to treat restless legs, restless leg syndrome can become a prominent and difficult feature of late-stage Parkinson's disease. It may have something to do with the rapid wearing off of levodopa with time, producing a withdrawal state.

\section{Early-Morning Dystonia and End-of-Dose Dystonia}

Early-morning dystonia is a debilitating, painful dystonia of the foot. It usually occurs early in the morning, before the first dose of levodopa. It tends to occur when the patient is "Off". It depends, however, on a critically low level of dopamine. It is generally regarded as a late side effect. ${ }^{18}$

Classically, it affects one or both feet, which become painful, cramped and forcibly inverted with curling of the toes, although the great toe may be hyperextended. More generalized dystonia involving the whole leg is seen less frequently.
The dystonic foot posture first occurs in the morning just after the patient places his foot on the floor. It is usually self-limited and subsides over one to two hours whether the patient takes levodopa or not. It does not commonly recur that day though it may the next morning. Taking the first morning dose of levodopa some fifteen to thirty minutes before arising may abolish the phenomenon. ${ }^{19}$

Foot dystonia occurs most often in those patients who exhibit "On-Off" fluctuations. It may consistently occur at the end of each "On" phase; other areas besides the legs may be involved. These dystonias are characteristically painful.

\section{Peak-Dose Dystonia}

This is dystonia occurring during the middle of the "On" period. It may occur alone or with dyskinesias. It is less frequent than "Off" dystonia.

\section{Akinetic Freezing of Gait}

Disturbances of gait, such as start hesitation and intermittent freezing are frequent accompaniments of late stage Parkinson's disease. They appear to be related to impaired postural reflexes and are resistant to levodopa therapy.

Freezing may be present during other motor activities such as writing and speaking. Repetitive movements such as supination and pronation of the forearm can be done well in slow tempo but with faster repetition will easily become frozen. The patient can perform slow repetitive actions normally but faster ones produce freezing. A characteristic feature of Parkinson's disease, the patient performs repetitive actions at a particular speed, 5 to 5.5 $\mathrm{Hz}$, very close to the tremor frequency. It is as if there is a CNS generator forcing movements to that frequency. This disturbance of repetitive movement is a specific difficulty of rhythm generation except at this critical frequency. ${ }^{20}$

This freezing phenomenon may be distinct from levodopa complications per se and is more likely related to the advance of the disease. Increasing freezing phenomena may reflect a peak dose effect of levodopa, under certain circumstances. It can be difficult to determine which type of freezing the patient is experiencing. ${ }^{21}$

\section{On-Off Phenomenon}

Wearing off typically evolves from a slow to a more rapid process. Initially it may be predictable. On-Off constitutes a sudden onset of parkinsonism. As time advances, however, the sudden "Offs" become unpredictable and unrelated to doses of antiParkinsonian medication. These random "On-Offs" constitute one of the most disabling features of late disease. Some patients switch "On and Off" so rapidly that the term "yo-yo-ing" has been suggested. ${ }^{22}$ The independence from levodopa doses may be more apparent than real. There may be failures of absorption of a levodopa dose or a dietary amino acid competition at the blood brain barrier that leads to the failure of any one dose to reach the brain. These peripheral effects may lead to random presentation of dopamine to the striatum in doses ranging from the inadequate to the toxic.

The clinical manifestations of "On-Off" phenomenon are sudden transitions within five minutes to marked parkinsonism. Improvement is frequently preceded by stereotyped dyskinesia. Many patients have a stereotyped pattern of abnormal involuntary movements as a prelude to switching "On". Striking changes 
in mood may be seen in these patients together with cognitive fluctuations. ${ }^{23}$

It is important to understand that there may be different clinical manifestations in unilateral as opposed to bilateral Parkinson's disease. In the patient with unilateral disease, particularly the non-dominant side, the "Off" may be non-debilitating. In the patient with bilateral disease the patient may be confined to bed or chair during the sudden "Offs." These attacks may occur several times per day.

"On-Off" with peak dose effects may be due, in some cases, to a depolarizing block. Under these circumstances significant autonomic changes are also present. This mechanism has been disputed.

As Parkinson's disease advances, the effect of a single dose of levodopa becomes less reliable. Patients become fearful and desperate when they realize their situation.

As time advances, the fluctuations become more frequent and more abrupt. Response to treatment becomes less and less predictable and reliable, and the dyskinesia itself becomes more and more disabling.

\section{Drug Failure}

Sometimes a single dose of levodopa fails to turn the patient "On" at all. This problem tends to occur late in the afternoon and may be in part a manifestation of peripheral mechanisms, such as poor gastric emptying, or absorbed amino acid competition at the blood brain barrier.

\section{Psychiatric Complications}

There are a number of psychiatric complications of levodopa therapy. These may be enumerated:

1. Sleep Disturbances

Vocalizations

Nightmares

Insomnia

Sleep behaviour disorder

2. Hallucinations

3. Psychosis

Delusions

Paranoid psychosis

4. Dementia (This is a manifestation of the disease or some other process but it often underlies the other complications.)

5. Depression

6. Anxiety and panic attacks

\section{Sleep Disturbances}

Sleep disturbances are usually in the form of vocalizations, nightmares or insomnia. These become progressively more apparent as time advances and frequently are a prelude to the more dramatic psychiatric manifestations.

The nightmares probably come about as a direct effect of levodopa on the brain. It may be that as the nigral cell population continues to wane that levodopa is taken up by the serotoninergic system and thereby stimulates that system with the resultant nightmares and subsequent daytime hallucinations. ${ }^{24}$

It is likely that the insomnia contributes to the daytime fatigue so common in the Parkinsonian population. In some patients levodopa seems to be soporific. This may lead to excessive daytime sleepiness and a reversal of the sleep-wake cycle. In others the waning benefits of levodopa lead to "Off" periods through the night with stiffness and pain. These tend to wake the patients. Sometimes there is a failure of the levodopa to control tremor and the patient on retiring experiences a significant heightening of his rest tremor which may be of sufficient magnitude to prevent him from sleeping.

The sleep behaviour disorder can be dramatic with the patient seemingly acting out his dreams to the fear and consternation of the spouse who may in rare instances be injured.

The role played by levodopa, as opposed to the natural progression of the disease, remains controversial.

\section{Hallucinations}

The hallucinations induced by levodopa are usually nonthreatening and visual. ${ }^{25}$ Patients are alert and aware at the time that they have these. As time goes on, the patients may become accustomed to them, but some continue to find them frightening. At first they are often relatively simple images of insects or birds, but with time they become more complex and more real. It is not unusual to have patients see complex scenes populated by numerous people, both known and unknown. One woman patient of mine saw her whole family of fourteen in her living room, necessitating her preparation of a meal for the multitude.

Auditory hallucinations have recently been described in Parkinson's disease. ${ }^{26}$ They do not occur without visual hallucinations. They tend to occur repetitively and are mainly human voices. They are non-imperative, non-paranoid, and often incomprehensible. Cognitive impairment is common.

\section{Psychosis}

The psychosis associated with Parkinson's disease is frequently paranoid. They may express jealousy of their spouse, though other paranoid delusions may be manifest. Patients become extremely difficult to manage at home when they reach this state. They accuse their partner of unfaithfulness, begin to search for evidence to support their claims and become angry and irrational.

\section{Dementia}

Dementia of course is a known complication in some $20 \%$ or more of patients with Parkinson's disease. ${ }^{27,28}$ In some instances it may be a reflection of superimposed Alzheimer's disease or indeed there may be a rapid development of Parkinsonism and dementia coincidentally as a reflection of diffuse Lewy body disease. A drug-induced dementing type picture may occur though more frequently a more acute disturbance in mental function in the form of an encephalopathy is created. A Parkinsonian patient with dementia should have the other correctable causes of dementia at least considered.

When dementia is present it provides a major hurdle to the management of the motor manifestations of Parkinson's disease because whatever drug is chosen to improve the motor symptoms will aggravate the psychiatric complications.

\section{Depression}

Depression affects 40-60\% of patients with Parkinson's disease. ${ }^{21}$ It seems to be related to the duration of the disease though it may be an early feature. The mechanism of this depression has not been fully resolved. It may have different etiologies in different patients. 


\section{Anxiety and Panic Attacks}

Panic attacks are not uncommon in the Parkinsonian population and they may relate to the activities of dopamine on adrenergic endings centrally.

\section{MANAGEMENT}

\section{Principles of Management}

The principles of management involve the following:

1. Adjusting levodopa intake

2. The use of supplementary medications

3. The use of dopamine agonists

4. The use of COMT inhibitors

5. The use of MAO-B inhibitors

6. Night-time sedation

7. The relief of the psychotoxicity

8. The management of depression

9. The management of other Parkinson's disease complications

10. Surgical considerations

11. Non-pharmacologic management

\section{Adjusting Levodopa Intake}

Critical in the management of levodopa complications is the adjustment of the levodopa intake. Levodopa absorption must be optimized and a critical dose of levodopa established.

Absorption of levodopa from the gut is dependent on the size of meals, the use or non-use of antacids and whether or not other anti-Parkinsonian medications are being used, such as anticholinergics, which tend to slow gastric emptying. Parkinson's disease itself probably slows gut motility. Constipation results in a reflex slowing of gastric emptying. All of these may be factors which prevent the delivery of levodopa to the proximal small bowel, from which it is absorbed by an active process. Following its absorption into the blood stream it must then compete with other amino acids to cross the blood brain barrier. An active transport system is involved. High protein meals may impede the absorption of levodopa into the brain. ${ }^{29}$

In order to optimize levodopa absorption it is usually helpful to have the patient take levodopa before a meal. This results in better and more rapid absorption. Dissolving levodopa in a fizzy drink or in water with or without ascorbic acid can be helpful, particularly if one wants a more rapid effect. Protein shifts to the evening meal probably make a minimal contribution, but in certain patients it can be helpful. It may be just as reasonable to space the protein intake evenly through the day. Stopping anti-cholinergics can be a useful strategy and sometimes levodopa absorption and anti-Parkinsonian effect can be improved by the addition of cisapride or domperidone, thereby increasing gastric motility. ${ }^{30}$

When fluctuations remain a significant problem, it can be helpful to establish a so-called critical dose of levodopa. One wants to give a single dose of levodopa first thing in the morning and follow the patient through the time course of the dose. It has been shown that lower doses given more frequently are probably counter-productive. There are many reasons for this. The dose is delayed in its absorption and the patient is already taking the next medication before the first one is fully absorbed. This leads to quite marked fluctuations that become increasingly unpredictable.
It has been suggested that the thresholds for dyskinesia and motor benefit are almost identical as the disease advances. This poses a significant problem with the use of levodopa as the only management for the late stages of the disease. None the less, a critical dose can sometimes be established, giving the minimum amount of dyskinesia and the maximum amount of motor benefit. That is the critical dose to be repeated at non-over-lapping intervals. As dyskinesia increases the dose may be spaced and predictable "Offs" accepted. If the patient is already on sustained action Sinemet, one should consider removing it and putting the patient on plain Sinemet. The rationale here is that the absorption of the plain Sinemet is more reliable. There are still people who use drug holiday siestas, skipping an afternoon dose in order to get a restoration of function later in the day. This strategy is used infrequently, but can be used successfully in certain patients.

Severe dyskinesia complicate late-stage Parkinson's disease. Patients often prefer these to the "Offs", but as time goes on, the dyskinesia become sufficiently severe to impair motor function. One strategy of management is a cautious levodopa reduction. Consideration might be given to a weekend or mid-afternoon socalled drug holiday siesta. These are used infrequently.

\section{The use of supplementary medications}

Amantadine is probably under utilized in late Parkinson's disease. It tends to be beneficial in smoothing out performance and lessens dyskinesia. ${ }^{31}$ Some have suggested a neuro-protective effect for this agent. It cannot be used in the face of significant cardiac disease, particularly with failure.

The early morning dystonia associated with the use of levodopa may respond to baclofen, lithium or an anti-cholinergic. Botox can sometimes be used in this context as well, with good success. ${ }^{32}$ Sometimes one need only give plain Sinemet a half hour before arising to offset early morning dystonia.

The myoclonus complicating Parkinson's disease will often respond to clonazepam.

Based on some anecdotal reports, I have had some good experience with the use of gabapentin for dyskinesia, allowing me to maintain levodopa at adequate levels for movement. ${ }^{33}$

\section{The Use of Dopamine Agonists}

The commonest strategy in managing the late complications of levodopa therapy, is the addition of an agonist. Currently, bromocriptine, pergolide, ropinirole and pramipexole are available.

There are certain principles in the use of agonist therapy. Even if one agonist has failed, it is worth trying the others. The peripheral side-effects which are similar to levodopa, including postural hypotension, nausea and vomiting, can be off-set by slow initiation of therapy and by the use of domperidone. Sometimes these agents, despite their pharmacology, are better used four times a day than three. They allow reduction of the dose of levodopa because the side-effect spectrum is slightly different and may depend upon different actions centrally. One can sometimes lessen the side-effects while maintaining optimum anti-Parkinsonian effects.

It is also important to be aware that the agents can be used in high doses, depending upon the patient's response. Clearly, if used beyond the manufacturers recommendation, it must be done 
with caution. It is wise to make the distinction between the ergotderived agonists and the newer agents. The latter can be used with greater safety, particularly in the elderly.

Significant postural hypotension and psychiatric complications will prevent the use of these agents.

\section{The Use of MAO-B Inhibition}

Selegiline is an MAO-B inhibitor and though this drug remains controversial, it does extend the action of dopamine and in consequence has a mild symptomatic effect. It sometimes can be useful in the late setting, but its benefits in advanced disease are minimal. Selegiline supplementing levodopa may reduce the incidence of dyskinesia. This is based on the principle that dopamine tends to work continuously at striatal endings. Because MAO-B inhibitors prevent the break-down of dopamine in the synaptic cleft they may enhance the duration of central action. ${ }^{34}$

There have been suggestions from the Parkinson's Disease Research Group of the United Kingdom that selegiline increases mortality in the parkinsonian population. ${ }^{35-37} \mathrm{~A}$ meta-analysis on five long-term, prospective, randomized trials of selegiline in patients with Parkinson's disease failed to confirm the United Kingdom findings. ${ }^{38}$

\section{The Use of COMT Inhibitors}

In the earlier stages when wearing off is the most significant factor, with minimal or no dyskinesia, a COMT inhibitor has significant benefit. There are two currently marketed: tolcapone and entacapone. Tolcapone ${ }^{39}$ inhibits catecholmethyltransferase peripherally and centrally, while entacapone ${ }^{40}$ appears to work peripherally. These drugs increase duration of action of levodopa, supposedly without increasing its maximum concentration in serum. It is important to remember to reduce the dose of levodopa by at least $25 \%$ coincident with the introduction of these agents. The benefits of these agents will be seen the next day.

Unfortunately, tolcapone (Tasmar) was associated with liver failure in a small number of patients and the agent has been withdrawn from the market in Canada.

\section{Night-time Sedation}

There are a number of nocturnal problems in Parkinson's disease. Because the patient goes for a relatively long period of time without medications through the night, wearing "Off" is very likely to occur in late disease. Night-time parkinsonism can cause severe sleep disturbance. Many Parkinsonians have autonomic deficiencies, particularly nocturia, which further disturb sleep with consequent day-time fatigue. Because righting reflexes are impaired, Parkinsonians often have a great deal of difficulty rising from bed to use the bathroom and often fall.

Night-time sleep can sometimes be benefited with amitriptyline. This drug has a sedative action, reduces urinary frequency, has a mild anti-Parkinsonian effect, as well as analgesic properties, but it blocks acetylcholine and should not be used in demented patients.

Slow release preparations, particularly Sinemet-CR, can sometimes get over some of the nocturnal difficulties and clonazepam hs can be useful for the myoclonic jerks. Cogentin has a long duration of action and can be used to prevent nighttime dys- tonia, urinary frequency and "Wearing off". The usual cautions apply to the use of anti-cholinergics.

\section{The Management of Psychotoxicity}

Psychotoxicity constitutes a major problem in the management of advanced Parkinson's disease, particularly when it has reached the point of a paranoid state or severe hallucinations. When it occurs it is clear that one should search for other causes of encephalopathy, particularly infections or other drug use. Normally, however, this complication requires the withdrawal of anti-Parkinsonian drugs, often progressively beginning with anticholinergics, selegiline and finally amantadine. Sometimes a limited drug holiday is of benefit.

Psychotoxicity can also be managed with certain medications. Clozapine can be used. This drug has beneficial effect on the psychosis associated with Parkinsonism and reducing hallucinations. It is an atypical neuroleptic acting as a D4 receptor antagonist and tends not to worsen the Parkinson's disease. ${ }^{41}$ The drug causes postural hypotension, sedation, delirium and sialorrhoea. The main difficulty with the drug is agranulocytosis. It requires weekly blood tests.

Olanzapine is probably less beneficial but sometimes is of help in psychotoxicity, particularly when depression is a component. Risperidone has been toted to be of value, but most clinicians would agree that this tends to make patients more Parkinsonian, and is best avoided. ${ }^{42}$

Ondansetron, a 5-HT3 receptor antagonist, is an expensive drug with which I have no personal experience. There is anecdotal evidence in the literature that it can be of benefit in the psychotoxicity associated with levodopa in late Parkinson's disease. ${ }^{43}$

More recently, quetiapine has been found to be extremely useful in the management of levodopa-induced psychoses. ${ }^{44}$

Also, it should be kept in mind that there is a fluctuating psychotoxicity associated with motor fluctuations. This leads to mood swings, anxiety when the medication wears off and transient cognitive dysfunction. The management is to improve the motor fluctuations.

\section{The Management of Depression}

Parkinson's disease patients with depression will usually respond to treatment with conventional antidepressants. ${ }^{26}$ Sedating antidepressants, such as amitriptyline, are useful for depressed patients with sleep disturbances. Stimulating antidepressants, such as fluoxetine, aid the depressed patient with predominant apathy. Doses are the same in Parkinson's disease as they are in the general population of depressed patients. The tricyclics tend to have anticholinergic effects and may aggravate dementia and the autonomic symptoms of Parkinson's disease. The selective serotonin reuptake inhibitors may interact with selegiline.

\section{The Management of Supplementary Problems}

There are other problems in Parkinson's disease which often have to be addressed. Sometimes dealing with them can reduce the late stage complications. Neurogenic bladder in the disease leads to nocturia, frequent awakenings, day-time fatigue and a worsening of the mental state. Ditropan can be helpful in relieving these night-time symptoms, but again should be used with some caution. 
Constipation is not only a perennial and chronic problem in the Parkinsonian disease population, but it frequently leads to delay in gastric emptying and worsens the patient's fluctuations. The usual techniques of exercises, fruits, fluids and bran should be employed. Senecot or glycerine suppositories may prove helpful and cisapride has some benefits. Botulinum Toxin Type A has been used with moderate success to control constipation resulting from puborectalis dyssynergia. ${ }^{45}$

Orthostatic hypotension is important to recognize. Failure to do so may result in significant harm to patients who may black out on arising to an up-right position. Its management initially might simply involve an increase of salt and adequate fluid intake. The head up-tilt at night is sometimes helpful. It is important to reduce nocturia because patients arising from a warm bed are most subject to this complication. Domperidone can be helpful. Unfortunately, sometimes it is necessary, though not often, to introduce fludrocortisone or Amatine to manage this complication.

\section{Surgery}

Finally, in late-stage disease, the resistant fluctuations and dyskinesia may require surgical intervention. It is beyond the scope of this article to discuss surgical management.

\section{Non-pharmacologic Management}

Patient well-being can often be improved by non-drug therapies in late Parkinson's disease. Appropriate dietary management, a regular and specifically designed exercise program, psychotherapy and emotional support for family and patient are important components of management.

\section{Conclusions}

Parkinson's Disease arises from the death of neurons in the substantia nigra. The cause of their death is unknown. Replacement of dopamine transmission with levodopa improves the symptoms of Parkinson's Disease. The mechanism of benefit is speculative and may not be simply pre-synaptic replenishment in nigral neurons. As time advances, the benefits of levodopa wane. Levodopa failure causes a sequence of well-defined clinical phenomena. The reason for these manifestations is debated. Our current strategies, in many instances, are inadequate to deal with these motor complications. Late-stage levodopa therapy is frequently complicated by neuro-behavioural complications. We are currently far removed from the final resolution in the medical management of late-stage Parkinsonism.

\section{REFERENCES}

1. Cotzias GC, Papavasiliou PS, Gellene R. Modification of Parkinsonism - chronic treatment with L-dopa. N Engl J Med 1969: 280: 337-345.

2. Stern MB. Contemporary approaches to the pharmacotherapeutic management of Parkinson's disease: an overview. Neurology 1997; 49 (Suppl 1): S1-S9.

3. Marsden CD. Parkinson's disease. J Neurol Neurosurg Psychiatry 1994; 57: 672-681.

4. Block G, et al. Sinemet-CR first, Eur Neurol 1997: 37: 93-97.

5. Miyawaki E, Lyons K, Pahwa R, Koller W. Motor complications of chronic levodopa therapy in Parkinson's disease. Clin Neuropharmacol 1997; 20: 523-530.

6. Marsden CD, Parkes JD, Quinn N. Fluctuations of disability in Parkinson's disease- clinical aspects. In: Marsden CD, Fahn S, eds. Movement Disorders. London: Butterworth Scientific, 1981: 96-122.
7. Rajput AH, Fenton Me, Birdi S, et al. Is levodopa toxic to human substantia nigra? Mov Disord 1997; 12(5): 634-638.

8. Rajput AH, Utti R, Rajput A, Offord K. Timely levodopa administration prolongs survival in Parkinson's disease. Parkinsonism Rel Disord 1997; Volume 3: 159-165.

9. Agid, Y. Levodopa: is toxicity a myth? Neurology $1998 ; 50(4)$ : 858863.

10. Rinne UK. Early combination of bromocritptine and levodopa in the treatment of early Parkinson's disease: a five year follow-up. Neurology 1987; 37: 826-828.

11. Fabbrini G, Juncos J, Mouradian MM, et al. Levodopa pharmacokinetic mechanisms and motor fluctuations in Parkinson's disease. Ann Neurol 1987; 21: 370-376.

12. Shaw KM, Lees AJ, Stern GM. The impact of treatment with levodopa on Parkinson's disease. QJ Med 1980: 49: 283-293.

13. Spencer SE, Wooten GF. Altered pharmacodynamics of L-dopa metabolism in rat striatum deprived of dopaminergic innervation. Neurology 1984: 34: 1105-1108.

14. Leenders, KL, Palmer AJ, Quinn N, et al. Brain dopamine metabolism in patients with Parkinson's disease measured with positron emission tomography. J Neurol Neurosurg Psychiatry 1986: 49: 853-860.

15. Fabbrini G, Mouradian MM, Juncos JL, et al. Motor fluctuations in Parkinson's disease: central pathophysiologic mechanisms. Ann Neurol 1988: 24: 366-371.

16. Stribe CMH, Kempster PA, Lees AJ, et al. Subcutaneous apomorphine in Parkinsonian on-off oscillations. Lancet 1988: 1: 403406.

17. Luquin MR, Scipioni O, Vaamonde J, et al. Levodopa-induced dyskinesias in Parkinson's disease: clinical and pharmacological classification. Mov Disord 1992; 7: 117-124.

18. Nausieda PA, Weiner WJ, Klawans HL. Dystonic foot response of Parkinsonism. Arch Neurol 1980: 37: 132-136.

19. Stoessl AJ. Prevention and management of late stage complications in Parkinson's disease. Can J Neurol Sci 1992; 19: 113-116.

20. Narabayashi H, Nakamura R. The freezing phenomenon-problems in long-term treatment for Parkinsonism. In: Rose FC, Capildeo $\mathrm{R}$, eds. Research Progress in Parkinson's Disease. Pitman Medical, 1981: 248-253.

21. Quinn NP. Classification of fluctuations in patients with Parkinson's disease. Neurology 1998; 51: S25-S29.

22. Marsden CD, Parkes JD. On-Off effects in patients with Parkinson's disease on chronic levodopa therapy. Lancet 1976: 1: 292-6.

23. Delis D, Direnfeld L, Alexander MP, et al. Cognitive fluctuations associated with on-off phenomenon. Neurology 1982: 32: 10491052.

24. Melamed E, Zoldan J, Friedberg G, et al. Involvement of serotonin in clinical features of Parkinson's disease and complications of Ldopa therapy. Adv Neurol 1996; 69: 545-550.

25. Factor SA, Molho ES, Podskalny GD, et al. Parkinson's disease: drug-induced psychiatric states. Adv Neurol 1995; 65: 115-138.

26. Inzelberg R, Kipervasser S, Korczyn AD. Auditory hallucinations in Parkinson's disease. J Neurol Neurosurg Psychiatry 1998; 64: 533-535.

27. Ebmeier KP, Calder SA, Crawford JR, et al. Dementia in idiopathic Parkinson's disease: prevalence and realtionship to the symptoms and signs of parkinsonism. Psychol Med 1991; 21: 69-76.

28. Tison F, Dartigues JF, Auriacombe S, et al. Dementia in Parkinson's disease: a population-based study in ambulatory and institutionalized individuals. Neurology 1995; 45: 705-708.

29. Melamed E, Bitton V, Zelig O. Episodic unresponsiveness to single doses of L-dopa in parkinsonian fluctuators. Neurology 1986; 36 : 100-103.

30. Djaldetti R, Koren M, Ziv I, et al. Effect of cisapride on response fluctuations in Parkinson's disease. Mov Disord 1995; 10: 81-84.

31. Verhagen Metman L, Del Dotto P, van den Munckhof P, et al. Amantadine as treatment for dyskinesia and motor fluctuations in Parkinson's disease. Neurology 1998; 50(5): 1323-1326.

32. Giladi N, Meer J, Honigman S. The use of botulinum toxin to treat striatal toes. J Neurol Neurosurg Psychiatry 1994; 57: 659.

33. Chana P, de Marinis A, Barrientos N. Letter to the editor. Mov Disord 1997; 12: 608 
34. Yahr M, Mendoza M, Moros D, et al. Treatment of Parkinson's disease in early and late phases: use of pharmacological agents with special reference to deprenyl (selegiline. Acta Neurol Scand 1983; (Suppl 95): 95-102.

35. Lees AJ. Comparison of therapeutic effects and mortality data of levodopa and levodopa combined with selegiline in patients with early, mild Parkinson's disease. Parkinson's Disease Research Group of the United Kingdom. Br Med J 1995; 311: 1602-1607.

36. Thorogood M, Armstrong B, Nichols T, Hollowell J. Mortality in people taking selegiline: observational study. Br Med J 1998; 317: 252-254.

37. Ben-Shlomo Y, Churchyard A, Head J, et al. Investigation by Parkinson's Disease Research Group of United Kingdom into excess mortality seen with combined levodopa and selegiline treatment in patients with early, mild Parkinson's disease: further results of randomised trial and confidential inquiry $\mathrm{Br}$ Med $\mathrm{J}$ 1998; 316: 1191-1196.

38. Olanow CW, Myllyla VV, Sotaniemi KA, et al. Effect of selegiline on mortality in patients with Parkinson's disease: a meta-analysis. Neurology 1998; 51: 825-830.

39. Limousin P, Pollak P, Pfeffen JP, et al. Acute administration of levodopa-benserazide and tolcapone, a COMT inhibitor, in Parkinson's disease. Clin Neuropharmacol 1995; 18: 258-265.
40. Merello M, Lees AJ, Webster R, et al. Effect of entacapone, a peripherally acting COMT inhibitor, on the motor response to acute treatment with levodopa in patients with Parkinson's disease. J Neurol Neurosurg Psychiatry 1994; 57: 186-189.

41. Rabey JM, Treves TA, Neufeld NY, Orlov E, et al. Low-dose clozapine in the treatment of levodopa-induced mental disturbances in Parkinson's disease. Neurology 1995; 45: 432-434.

42. Rich SS, Friedman JH, Ott BR. Risperidone versus clozapine in the treatment of psychosis in six patients with Parkinson's disease and other akinetic-rigid syndromes. J Clin Psych 1995; 56: 556559.

43. Zoldan J, Friedberg G, Weizman A, Melamed E. Ondansetron, a 5HT3 antagonist for visual hallucinations and paranoid delusional disorder associated with chronic levodopa therapy in advanced Parkinson's disease. Adv Neurol 1996; 69: 541-544.

44. Bastani B, Parsa MA. Quetiapine (Seroquel) in the treatment of psychosis in patients with Parkinson's disease. J Neuropsychiatry Clin Neurosci 1998; 10: 216-219.

45. Albanese A, Maria G, Bentivoglio AR. Severe constipation in Parkinson's disease relieved by botulinum toxin. Mov Disord 1997; 12: 764-766. 